\title{
Managing challenging behaviour in preschool children post-traumatic brain injury with online clinician support: protocol for a pilot study
}

Kaitlyn Taylor ${ }^{1,2^{*}}$ (D), Cathy Catroppa ${ }^{1,3,5}$, Celia Godfrey ${ }^{1,5}$, Audrey McKinlay ${ }^{3}$, Jennie Ponsford ${ }^{2}$, Jan Matthews ${ }^{6}$ and Vicki Anderson 1,3,4,5

\begin{abstract}
Background: Traumatic brain injury (TBI) in children is associated with a range of poor long-term outcomes, including behavioural disturbances. Parents can experience high levels of stress and injury-related burden, and evidence suggests that distressed parents are less likely to adopt positive parenting styles to manage their child's behaviour. The 'Signposts for Building Better Behaviour' program is a parenting programme that was originally developed to assist parents of children with an intellectual disability in managing their child's behaviour. More recently, it has been adapted to include a TBI module, to assist parents in managing post-TBI behaviour. However, geographical and financial barriers remain, preventing many parents from accessing the programme in the standard face-to-face modality. This project aims to investigate the feasibility and acceptability of the programme when delivered with clinician support via videoconferencing.

Methods/design: The sample for this feasibility study will be recruited from the Royal Children's Hospital, Melbourne, and the Victorian Paediatric Rehabilitation Service. Participants will be the parents of a child who sustained a TBI between the ages of 2.0 and 6.11 , within the previous 2 years. The parents of 15 children will complete the programme, with clinician support via videoconferencing, while the parents of a further 15 children will form a treatment as usual wait-list control group. Parents complete questionnaires assessing their child's behaviour, as well as assessing their own mental health, sense of parenting competency, disciplinary style, and family functioning. These will be completed upon enrolment in the study regarding their child's pre-injury behaviour and then again pre-intervention, immediately post-intervention, and 4 months post-intervention. Parents who complete the intervention will also complete questionnaires assessing their satisfaction with the programme and its delivery. Information will be collected on the feasibility, clinical practicality, and acceptability of the programme when delivered through this medium.
\end{abstract}

Discussion: This study is the first to investigate the feasibility of delivering post-child TBI behavioural intervention via videoconferencing in Australia. Preliminary findings from this study may support the development of a larger randomised controlled trial. It is hoped that programme delivery through this medium would facilitate better access to the programme, enabling improved long-term outcomes for families.

Trial registration: ANZCTR, ACTRN12616001574437

Keywords: Paediatric traumatic brain injury, Behaviour, Parenting programme, Intervention

\footnotetext{
* Correspondence: kaitlyn.taylor@mcri.edu.au

${ }^{1}$ Child Neuropsychology, Murdoch Childrens Research Institute, 50

Flemington Rd, Parkville, Victoria 3052, Australia

${ }^{2}$ Psychological Sciences, Monash University, Melbourne, Australia

Full list of author information is available at the end of the article
} 


\section{Background}

\section{Child behavioural outcomes post-TBI}

Paediatric traumatic brain injury (TBI) is a leading cause of disease burden in young children [1] and is associated with a range of poor psychological outcomes [2]. This includes post-injury behaviour changes [3], which can include excessive externalising behaviour, where the child demonstrates aggression, non-compliance, and irritability [4,5]. The child may also exhibit poor selfregulation [6] and behave inappropriately in social settings [7, 8]. Internalising behaviours may also be present, in which the child is withdrawn and anxious [3], and parents may report personality changes [9].

The prevalence of such changes have been documented in $36 \%$ of severe TBI cases and $22 \%$ of moderate TBI cases [10]. Research also suggests that young children who experience a mild TBI requiring inpatient care are more likely to have behaviour problems than their uninjured peers [11]. Indeed, Catroppa and colleagues [12] showed that behaviour problems can emerge regardless of injury severity. These changes have been demonstrated to emerge early post-injury and increase over time [10] and may persist in the long-term [10, 11]. Behaviour disturbances post-injury have the potential to set individuals on a poor long-term trajectory, which may include difficulties in the classroom [10], anti-social behaviour [4], and increased risk of criminality [13]. Thus, problematic behaviour post-TBI is an outcome which requires attention and cannot be assumed to resolve without intervention.

\section{Parent outcomes when caring for a child post-TBI}

Parental stress and family burden are also elevated following a child TBI. More specifically, behaviour disturbances post-TBI have been demonstrated to relate to higher parent distress and greater injury-related family burden [10]. Evidence suggests that this relationship is bi-directional, where parental distress, and mental health more generally, also uniquely predicts child behaviour problems [14]. Parents exhibiting greater distress are suggested to be less likely to adopt positive parenting styles to manage their child's behaviour [15]. Relatedly, poorer parental communication [16] and disciplinary practices [17] relate to poorer post-TBI outcomes. Therefore, interventions which aim to reduce parent distress, and improve parental disciplinary styles and family functioning, may be particularly beneficial in improving post-paediatric TBI behaviour.

\section{The Signposts for Building Better Behaviour programme} 'Signposts for Building Better Behaviour' (Signposts) is an intervention programme initially developed to assist parents in managing challenging behaviours in children with intellectual disability [18], with the additional aim of decreasing parent distress and increasing their sense of competence in managing their child's behaviour. Signposts is a manualised programme in which parents read modules, watch videos, and complete homework exercises. Drawing on principles from behavioural therapies such as positive behaviour support, the programme aims to teach parents to conduct a functional analysis of a target behaviour, then design and implement an intervention to replace this behaviour with more desirable behaviour. The role of the Signposts therapist is to reiterate key messages from the module, answer any questions that the parent may have, and troubleshoot homework difficulties. The programme was designed to be delivered in a variety of modes of delivery, including a group format, as well as with clinician support via telephone, or in a self-directed manner (i.e. without the support of a clinician). However, research has suggested that those who receive support from a clinician during the programme are more likely to successfully complete the full programme [19].

Signposts has more recently been adapted to be relevant for parents of children with a TBI, with the inclusion of an additional paediatric TBI psycho-education module [20]. The modified programme was found to have high levels of consumer satisfaction for the parents of children with a TBI, who found the modules and the skills taught to be relevant and useful [21]. Parents reported significant reductions in challenging child behaviours, as well as significant reductions in dysfunctional parenting practices, stress, and family burden, when completing the programme through both group support and telephone support practices [22]. Evidence suggests good maintenance of these effects in the longterm post-intervention [23].

\section{The use of technology in delivering health interventions}

Electronic health services, or 'eHealth', commonly refer to the 'use of information technology in the delivery of health care' [24]. Several studies report efficacy in the use of eHealth as a medium for delivering post-TBI behaviour interventions to families, including research utilising psycho-education and web-based family-oriented therapy, with associated improvements in caregiver distress [25-27].

The use of the Internet to deliver therapy for postpaediatric TBI behavioural disturbance has many benefits. Videoconferencing may be beneficial as parents would not require transport or childcare and could potentially avoid time taken from work to attend faceto-face therapy sessions [28]. Paediatric TBI causes a considerable degree of financial strain for families [29], and any method for alleviating this strain is worth investigating. Furthermore, the risk of sustaining a paediatric TBI is increased for those who live in remote areas [30], 
highlighting the need to increase access to interventions for those who live far from rehabilitative services.

While receiving telephone support for post-TBI rehabilitation can assist in removing some of these socioeconomic and geographical barriers, videoconferencing has several additional benefits [31]. Video technology means improved ability to detect non-verbal cues and provides an opportunity for including more than just one parent in the session. It enables shared viewing of programme materials such as videos, graphics, and homework exercises-none of which is possible via a traditional telephone. While videoconferencing is relatively new to post-paediatric TBI care, research in the related area of mental health intervention reports that videoconferencing has a comparable level of efficacy when compared to standard face-to-face delivery. For example, one randomised controlled trial comparing therapy across these modes of delivery found that posttherapy, $74.3 \%$ of patients treated via videoconferencing showed a reliable improvement in symptoms, compared to $75 \%$ when treated face to face [32]. Further, researchers have found there to be no significant difference between the two modes of delivery regarding the quality of the therapeutic alliance when rated by the client $(p=0.53)$ or the therapist $(p=0.60)$, or regarding client ratings of service satisfaction $(p=0.77)$ [33]. Several studies have reported that videoconferencing is more cost effective than standard face-to-face care delivery [34].

\section{Study aims}

The primary aim of the current study is to investigate the feasibility and acceptability of conducting Signposts with videoconferencing as the mode of clinician support. In particular, this study intends to investigate this in families where the child sustained the TBI in early childhood, an age group that may be particularly prone to post-TBI poor outcomes [35-37] but, to date, has been relatively neglected with minimal attention directed to development of age-appropriate evidence-based interventions targeting behaviour problems.

More specifically, the study objectives are to:

1. Investigate the feasibility of delivering the programme with clinician support via videoconferencing, with the goal of developing a larger RCT. This will be investigated with the collection of data on sample retention rates, participant programme adherence, and duration to reach target sample size.

2. Collect information to inform on the clinical practicality of delivery through this medium, including the duration of videoconference sessions, rate of technology difficulties that cause disruption to sessions, and the time frame for programme completion (in weeks).
3. Explore the acceptability of the programme when delivered through this medium, with completion of a consumer satisfaction survey, as well as by enquiring on the participant's comfort with using the technology. It is expected that, consistent with programme delivery through different modalities, the programme will have a high level of consumer satisfaction in this population when delivered with clinician support via videoconferencing.

4. Explore preliminary clinical outcomes across those who completed the programme compared with those on a wait-list, in order to inform likely efficacy estimates for a fully powered RCT. Primary measures will assess child behaviour, while secondary outcome measures will assess parent distress, parenting disciplinary style, parenting sense of competency, and family functioning. These will be measured using questionnaires considered standard for research involving the Signposts programme (see, for example, Woods and colleagues [23]). It is expected that those who complete Signposts will report reductions in post-TBI child behaviour problems, parental distress, and dysfunctional parenting, while increasing family functioning and parental sense of competency.

\section{Methods/design}

\section{Overall study design}

The current pilot study employs a two-arm, parallel non-randomised design, in which a treatment as usual wait-list group is compared with a group in which the 'Signposts' programme is completed with clinician support via videoconferencing (see Fig. 1). The wait-list group may access any therapies or treatments considered standard treatment for a paediatric TBI.

Data will be collected at four time points: (i) preinjury functioning at the time of enrolment in the study and then (ii) pre-intervention, (iii) immediately postintervention, and (iv) at the 4-month follow-up postintervention. Data collected from those who complete the intervention will be compared with data collected at equivalent time points from a wait-list control group. This study has been granted ethics approval by the Human Research Ethics Committee of the Royal Children's Hospital, Melbourne.

\section{Participants}

Participants are the primary caregivers (herein referred to as parents) of at least one child who has sustained a TBI within the prior 2 years. Children aged 2.0-6.11 at the time of injury, who sustained mild, moderate, or severe injuries, will be eligible to participate.

Inclusion criteria for the current study are that medical records suggest that parents and children have sufficient English skills, that the child has no prior 


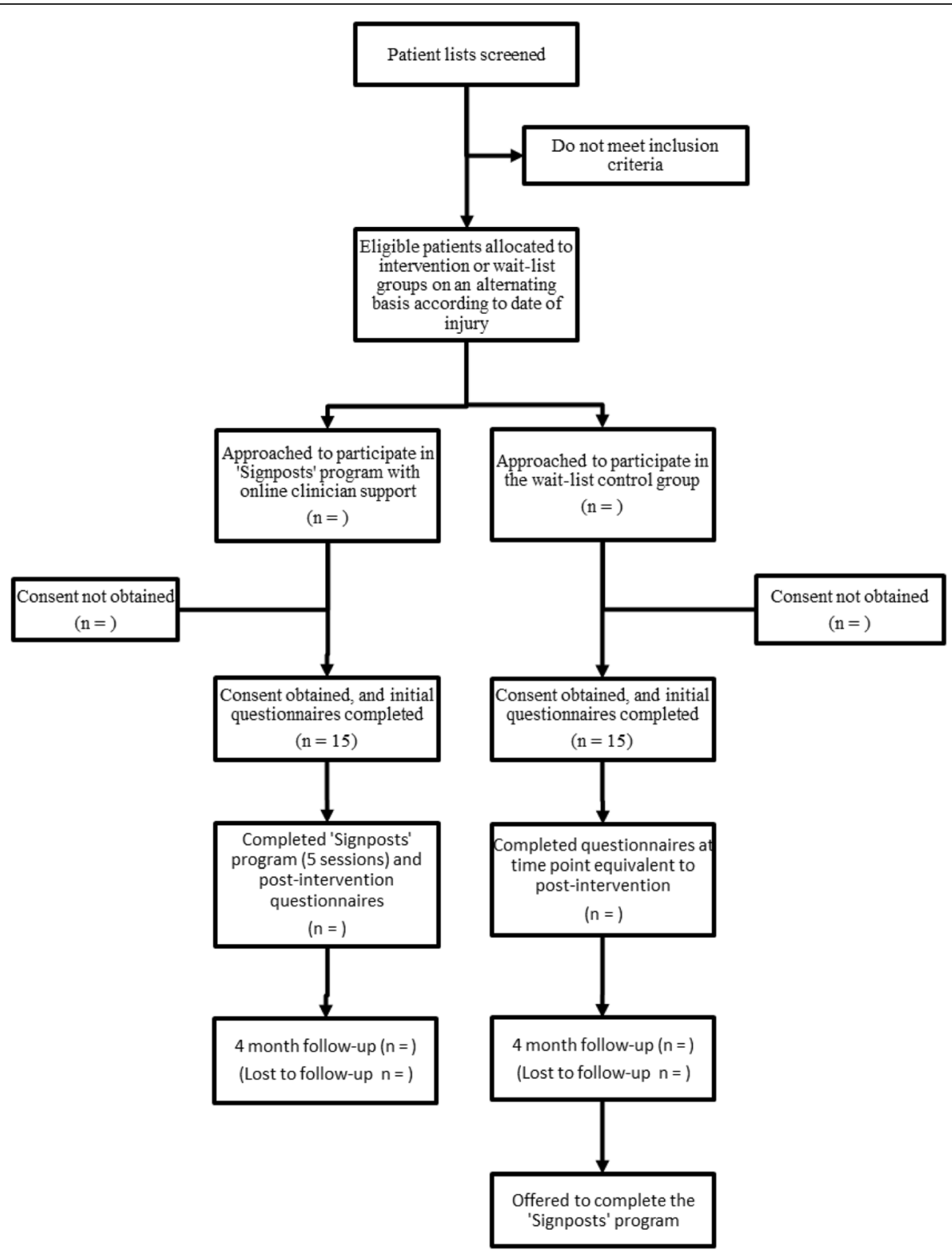

Fig. 1 Participant flow diagram

history of neurological or developmental disorder, no diagnosed severe psychiatric disorder in the parent, and no documented evidence that the TBI was nonaccidental.

In keeping with previous research [38], TBI will be classified by injury severity (mild, moderate, or severe). For young children, these categories are largely based on scores on the Paediatric Glasgow Coma Scale (GCS) [39], which provides ratings of the depth and/or duration of altered consciousness post-injury. As an adaptation to the initial scale devised for use in adults [40], GCS is instead rated in young children by recording behavioural rather than verbal responses. Scores are assigned on a scale of $3-15$, where 3 indicates no verbal, motor, or visual responsiveness to stimuli, and 15 indicates full responsiveness.

Classifications of injury severity are made according to the following criteria:

(i) Mild: GCS 13-15, no evidence of mass lesion on CT/MRI and no neurological deficits

(ii) Moderate: GCS 9-12, mass lesion or other evidence of specific injury on CT/MRI and/or neurological impairment 
(iii)Severe: GCS 3-8, mass lesion or other evidence of specific injury on CT/MRI and/or neurological impairment

\section{Measures \\ Injury and demographic variables}

Injury and medical characteristics of the injured child are obtained, including lowest GCS, length of coma, neuroimaging abnormalities, injury aetiology, family structure, ethnicity, parental education, and parental occupation. Social adversity is calculated based on the Social Risk Index (SRI), which is comprised of six aspects of social status and provides an aggregate score, where a score of 0 or 1 denotes low social adversity, and a score of $>2$ denotes high social adversity [41].

\section{Child behavioural outcomes}

The pre-morbid, pre-intervention, and post-intervention behavioural functioning of the children is determined using the Child Behavior Checklist (CBCL) [42]. Two versions of the CBCL will be used. The preschool version of the CBCL is a standardised 100-item questionnaire, which was designed for use on children aged 1.5-5, while the school-aged version is a 113-item questionnaire designed for use on children aged 6-18. The age-appropriate version will be completed by the caregiver regarding their child's behaviour. The current study used the three summary scores for analysis (total, internalising and externalising behaviour scales). $T$ scores are derived for these scales $(M=50, \mathrm{SD}=10)$, where a $T$ score greater than or equal to 60 denotes behaviour falling in the clinically significant range.

\section{Family outcomes}

Family functioning will be assessed with the self-report McMaster Family Assessment Device (FAD) [43, 44]. The 12-item summary scale, FAD-General Functioning (FAD-GF), will be used for analysis. High family dysfunction is indicated by a mean FAD-GF score $>2.17$ [45].

\section{Parental functioning}

Parental psychological distress will be measured using the Depression Anxiety Stress Scales (DASS) [46]. The DASS is a 42-item self-report inventory designed to measure the negative emotional states of depression, anxiety, and stress. Symptom levels on each subscale are categorised as falling in the normal, mild, moderate, severe, and extremely severe range according to the criteria outlined in Table 1.

The total for each subscale will be used for analysis in the current study.

The Parenting Scale (PS) will be used to measure different styles of disciplinary practices [47]. The PS is a 30 -item questionnaire that yields three measures of
Table 1 Subscale severity criteria for the DASS

\begin{tabular}{llll}
\hline Severity & Depression & Anxiety & Stress \\
\hline Normal & $0-9$ & $0-7$ & $0-14$ \\
Mild & $10-13$ & $8-9$ & $15-18$ \\
Moderate & $14-20$ & $10-14$ & $19-25$ \\
Severe & $21-27$ & $15-19$ & $26-33$ \\
Extremely severe & $28+$ & $20+$ & $34+$ \\
\hline
\end{tabular}

Lovibond and Lovibond [46]

dysfunctional disciplinary styles in parents: laxness, overreactivity, and verbosity. A total dysfunctional disciplinary style score can be calculated where lower scores reflect better parenting. This score will be used for analysis in the current study.

The Parenting Sense of Competence Scale (PSOC) will be used to measure the parent's self-reported competency and satisfaction in their parenting [48]. The scale consists of 16 items, from which two dimensions can be derived, reflecting satisfaction with their parenting role and sense of self-efficacy in the parenting role. Total scores across these two dimensions will be used for analysis, with higher scores reflecting greater satisfaction and efficacy.

\section{Programme satisfaction}

Parent satisfaction with the Signposts programme, and its delivery, will be measured using the nine-item Consumer Satisfaction Scale (CSS), which was developed for the purpose of investigating satisfaction with the Signposts programme [18]. Items on this measure are recorded on a five-point Likert scale, in which participants indicate their level of agreement with a statement about their experience with the programme, from 'Strongly Disagree' to 'Strongly Agree'.

\section{Procedure \\ Recruitment}

Eligible parents are identified by clinicians working in relevant departments at the Royal Children's Hospital and the Victorian Paediatric Rehabilitation Service in Melbourne. Parents will then be allocated to either the intervention or wait-list control group on an alternating basis according to date of injury.

Recruitment letters will be mailed to parents, introducing the study and inviting them to participate. Parents are provided with information statements and sign the consent form should they wish to participate. Parents who do not accept or decline participation within 2 weeks will be followed up with a phone call. 


\section{Data collection}

Data will be recorded to capture the number of eligible parents approached, the number who consented to the programme, and the number who successfully completed the programme, as well as reasons for nonparticipation and non-completion.

Questionnaires will be administered using a secure online data collection instrument (RedCap), with parents sent web links at the appropriate time points. Upon enrolment in the study, parents will be sent the web-link questionnaires and asked to complete the outcome measure questionnaires retrospectively, reporting on their child and family's functioning prior to the TBI. It is acknowledged that this may be vulnerable to parent reporting bias, particularly where children are several years post-injury. However, evidence suggests that postTBI behavioural problems may not emerge or present for intervention until several years post-injury [10, 11], which means that including families several years postinjury more closely mirrors the clinical setting. It therefore seems reasonable to include such families in this feasibility study. Future research may aim to minimise this bias by reducing the eligible time since injury.

Prior to commencing the programme, approximately 1 month following initial questionnaire completion, parents will be asked to complete the measures again with regard to their family and child's current functioning. Outcome questionnaires will again be sent in the form of web links via email immediately after the intervention and 4 months post-intervention. The approximate timeline for completion of these questionnaires is summarised in Table 2.

Table 2 Summary of measures used

\begin{tabular}{|c|c|c|c|c|}
\hline Construct & Measure & Time & e po & \\
\hline & & 1 & 23 & 3 \\
\hline Socioeconomic status & Social Risk Index & $\bullet$ & & \\
\hline Outcome measures & & & & \\
\hline Child behavioural outcomes & Child Behavior Checklist & $\bullet$ & • & • \\
\hline Family outcomes & $\begin{array}{l}\text { McMaster Family } \\
\text { Assessment Device }\end{array}$ & & & • \\
\hline Parental functioning & & & & \\
\hline Parental psychological distress & $\begin{array}{l}\text { Depression Anxiety } \\
\text { Stress Scales }\end{array}$ & & & • \\
\hline $\begin{array}{l}\text { Confidence and satisfaction } \\
\text { in the parenting role }\end{array}$ & $\begin{array}{l}\text { Parenting Sense of } \\
\text { Competence Scale }\end{array}$ & & & \\
\hline Disciplinary practices & Parenting Scale & & $\bullet$ & • \\
\hline Programme satisfaction & $\begin{array}{l}\text { Consumer Satisfaction } \\
\text { Scale }\end{array}$ & & & \\
\hline
\end{tabular}

Time point $1=$ retrospectively completed about pre-injury behaviour, $2=$ preintervention, 3 = post-intervention, $4=4$ months post-intervention

antervention group only

\section{Intervention procedure}

Prior to commencement of this study, the Signposts clinician will complete the Professional Training Workshop through the Parenting Research Centre in Melbourne, where the original Signposts programme was developed. Workbooks and modules used in the current study are in the original hard copy form, available through the Parenting Research Centre. In addition, this project uses the stand-alone adjunct module 'Dealing with a head injury in the family', developed by Woods and colleagues [20].

Children must be at least 3 months post-injury before their parents commence the programme. When ready to commence the programme, participants engage in an initial face-to-face interview with the Signposts clinician, which provides the opportunity to build rapport and further explain the requirements of the programme, including demonstration of the iPads and the videoconferencing software. Initial modules required to begin the programme $(1-3,8$, and 9$)$ are provided at this session. The programme is commenced after this initial interview.

Parents work through module booklets in their own time and complete the relevant tasks in their workbook. On five occasions, approximately 2 weeks apart, parents engage in a one-on-one videoconference session with the Signposts clinician. The session follows the suggested structure for telephone support from the practitioner manual, which generally aims to support parents in completing homework and answer any questions or concerns that may arise.

In line with previous research [21], some programme modules were grouped together for logistical purposes. The approximate timeline for the completion of modules and videoconference sessions is presented in Table 3. Aside from the initial modules provided at the preintervention interview, subsequent modules (4-7) are individually sent to the family via post, as needed.

\section{Sample size}

The current feasibility study sample will aim to consist of 15 parents who will participate in the intervention and a further 15 allocated to the wait-list control group. Studies also exploring the feasibility of family-centred behaviour interventions in this population used similar sample sizes [49-52] and detected promising preliminary results. With recruitment in this population frequently difficult and slow, we aim to measure the duration of time taken to recruit this sample in order to guide estimates of recruitment duration for a larger RCT.

\section{Data analysis}

Frequencies and percentages will be used to describe the population across key demographic and injury variables. We will compare the intervention and wait-list control 
Table 3 Programme for completion of Signposts programme with online clinician support

\begin{tabular}{|c|c|c|}
\hline Week & Parent homework & Clinician contact \\
\hline 0 & & Pre-intervention interview \\
\hline 1 & $\begin{array}{l}\text { Read modules } 1-3,8 \text {, and } 9 \\
\text { Begin homework }\end{array}$ & \\
\hline 2 & $\begin{array}{l}\text { Complete homework for modules } \\
1-3,8 \text {, and } 9 \text { prior to } \\
\text { videoconference }\end{array}$ & $\begin{array}{l}\text { Videoconference \#1 } \\
\text { Homework reviewed for } \\
\text { modules } 1-3,8 \text {, and } 9\end{array}$ \\
\hline 3 & $\begin{array}{l}\text { Read module } 4 \\
\text { Begin homework }\end{array}$ & \\
\hline 4 & $\begin{array}{l}\text { Complete homework for module } \\
4 \text { prior to videoconference }\end{array}$ & $\begin{array}{l}\text { Videoconference \#2 } \\
\text { Homework reviewed for } \\
\text { module } 4\end{array}$ \\
\hline 5 & $\begin{array}{l}\text { Read module } 5 \\
\text { Begin homework }\end{array}$ & \\
\hline 6 & $\begin{array}{l}\text { Complete homework for module } \\
5 \text { prior to videoconference }\end{array}$ & $\begin{array}{l}\text { Videoconference \#3 } \\
\text { Homework reviewed for } \\
\text { module } 5\end{array}$ \\
\hline 7 & $\begin{array}{l}\text { Read module } 6 \\
\text { Begin homework }\end{array}$ & \\
\hline 8 & $\begin{array}{l}\text { Complete homework for module } \\
6 \text { prior to videoconference }\end{array}$ & $\begin{array}{l}\text { Videoconference \#4 } \\
\text { Homework reviewed for } \\
\text { module } 6\end{array}$ \\
\hline 9 & $\begin{array}{l}\text { Read module } 7 \\
\text { Begin homework }\end{array}$ & \\
\hline 10 & $\begin{array}{l}\text { Complete homework for module } \\
7 \text { prior to videoconference }\end{array}$ & $\begin{array}{l}\text { Videoconference \#5 } \\
\text { Homework reviewed for } \\
\text { module } 7\end{array}$ \\
\hline 11 & $\begin{array}{l}\text { Complete post-intervention } \\
\text { questionnaires } \\
\text { Return iPad }\end{array}$ & \\
\hline
\end{tabular}

groups across key variables (age at injury, injury severity, social risk, pre-injury behaviour, and pre-intervention behaviour) to detect any significant difference which may later compromise the generalisation of the findings.

Investigating the feasibility, clinical practicality, and acceptability of Signposts with videoconferencing support will be exploratory in nature. The objective is to collect information on ease of use, participant satisfaction, homework completion rates, programme completion and adherence, and attrition rates. Rates of programme attrition will be reported, as well as consumer satisfaction with the programme (reported as mean scores and percentages for items on the CSS).

To examine preliminary data on the impact of the programme on key outcome areas, we will compare the post-intervention scores on the CBCL scales, FAD, DASS, PS, and PSOC across the intervention and control groups and will report the mean and standard deviation of each group across these measures. To report preliminary findings on the maintenance of any treatment effects, means and standard deviations will be reported to assess any change within the intervention group from postintervention to 4-month follow-up across the CBCL scales,
FAD, DASS, PS, and PSOC. These findings may inform efficacy estimates for a larger RCT.

\section{Discussion}

This study is the first to investigate the feasibility and efficacy of a parenting training programme using videoconferencing as the support mode, for decreasing undesirable behaviours in children who sustained a TBI at an early age and improving parental post-TBI outcomes, such as mental health and sense of parenting efficacy. Preliminary findings of feasibility and efficacy of Signposts when delivered with an online support mode will pave the way for use of this method in a larger randomised controlled trial, with the aim of translation into clinical settings. For many families, when implemented clinically, online programme delivery would provide the opportunity to access services which would otherwise be costly and time consuming, particularly for those from more challenging socioeconomic backgrounds, or living in rural settings. This initial study also lays the foundation to further develop Signposts materials to be fully provided online.

\section{Abbreviations \\ CBCL: Child Behavior Checklist; CSS: Consumer Satisfaction Scale; DASS: Depression Anxiety Stress Scale; FAD: Family Assessment Device; FAD- GF: Family Assessment Device-General Functioning; GCS: Glasgow Coma Scale; PS: Parenting Scale; PSOC: Parenting Sense of Competence Scale; SRI: Social Risk Index; TBI: Traumatic brain injury}

\section{Funding}

This research will be funded by the Victorian Government Operational Infrastructure Scheme.

\section{Availability of data and materials}

Not applicable - this manuscript outlines a protocol.

\section{Author's contributions}

All authors contributed to the writing of this manuscript, as well as contributed to the conception and design of this study. All authors read and approved the final manuscript.

\section{Competing interests}

The authors declare that they have no competing interests.

\section{Consent for publication}

Not applicable.

\section{Ethics approval and consent to participate}

This research received ethics approval from the Human Research Ethics Committee of the Royal Children's Hospital, Melbourne (HREC 24011). Informed consent is obtained from all participants.

\section{Publisher's Note}

Springer Nature remains neutral with regard to jurisdictional claims in published maps and institutional affiliations.

\section{Author details}

${ }^{1}$ Child Neuropsychology, Murdoch Childrens Research Institute, 50 Flemington Rd, Parkville, Victoria 3052, Australia. ${ }^{2}$ Psychological Sciences, Monash University, Melbourne, Australia. ${ }^{3}$ Psychological Sciences, University of Melbourne, Melbourne, Australia. ${ }^{4}$ Psychology Service, Royal Children's Hospital, Melbourne, Australia. ${ }^{5}$ Department of Paediatrics, University of Melbourne, Melbourne, Australia. ${ }^{6}$ Parenting Research Centre, Melbourne, Australia. 
Received: 16 November 2016 Accepted: 3 May 2017 Published online: 31 May 2017

\section{References}

1. Fletcher JM, Ewing-Cobbs L, Francis DJ, Levin HS. Variability in outcomes after traumatic brain injury in children: a developmental perspective. In Traumatic head injury in children. Edited by Broman S, Michel ME. New York: Oxford University Press; 1995:3-21.

2. Babikian T, Asarnow R. Neurocognitive outcomes and recovery after pediatric TBI: meta-analytic review of the literature. Neuropsychology. 2009; 23:283.

3. Asarnow RF, Satz P, Light R, Lewis R, Neumann E. Behavior problems and adaptive functioning in children with mild and severe closed head injury. J Pediatr Psychol. 1991;16:543-55.

4. Andrews T, Rose F, Johnson D. Social and behavioural effects of traumatic brain injury in children. Brain Inj. 1998;12:133-8.

5. Cole WR, Gerring JP, Gray RM, Vasa RA, Salorio CF, Grados M, Christensen JR, Slomine BS. Prevalence of aggressive behaviour after severe paediatric traumatic brain injury. Brain Inj. 2008;22:932-9.

6. Ganesalingam K, Sanson A, Anderson V, Yeates KO. Self-regulation and social and behavioral functioning following childhood traumatic brain injury. J Int Neuropsychol Soc. 2006;12:609-21.

7. Rutter M, Chadwick O, Shaffer D. Head injury. In Developmental Neuropsychiatry. Edited by Rutter M. New York: Guilford Publications, Incorporated; 1983.

8. Rosema S, Crowe LM, Anderson V. Social function in children and adolescents after traumatic brain injury: a systematic review 1989-2011. J Neurotrauma. 2012;29:1277-91.

9. Max JE, Robertson BAM, Lansing AE. The phenomenology of personality change due to traumatic brain injury in children and adolescents. J Neuropsychiatry Clin Neurosci. 2001;13:161-70.

10. Schwartz L, Taylor HG, Drotar D, Yeates KO, Wade SL, Stancin T. Long-term behavior problems following pediatric traumatic brain injury: prevalence, predictors, and correlates. J Pediatr Psychol. 2003;28:251-63.

11. McKinlay A, Grace R, Horwood L, Fergusson D, MacFarlane M. Long-term behavioural outcomes of pre-school mild traumatic brain injury. Child Care Health Dev. 2010;36:22-30.

12. Catroppa C, Godfrey C, Rosenfeld JV, Hearps SS, Anderson VA. Functional recovery ten years after pediatric traumatic brain injury: outcomes and predictors. J Neurotrauma. 2012;29:2539-47.

13. Williams WH, McAuliffe KA, Cohen MH, Parsonage M, Ramsbotham J. Traumatic brain injury and juvenile offending: complex causal links offer multiple targets to reduce crime. J Head Trauma Rehabil. 2015;30:69-74.

14. Taylor HG, Yeates KO, Wade SL, Drotar D, Stancin T, Burant C. Bidirectional child-family influences on outcomes of traumatic brain injury in children. J Int Neuropsychol Soc. 2001;7:755-67.

15. Woods D, Catroppa C, Barnett P, Anderson VA. Parental disciplinary practices following acquired brain injury in children. Dev Neurorehabil. 2011;14:274-82.

16. Raj SP, Wade SL, Cassedy A, Taylor HG, Stancin T, Brown TM, Kirkwood MW. Parent psychological functioning and communication predict externalizing behavior problems after pediatric traumatic brain injury. J Pediatr Psychol. 2014;39:84-95.

17. Micklewright JL, King TZ, O'Toole K, Henrich C, Floyd FJ. Parental distress, parenting practices, and child adaptive outcomes following traumatic brain injury. J Int Neuropsychol Soc. 2012;18:343-50.

18. Hudson A, Matthews J, Gavidia-Payne S, Cameron C, Mildon R, Radler G, Nankervis K. Evaluation of an intervention system for parents of children with intellectual disability and challenging behaviour. J Intellect Disabil Res. 2003:47:238-49.

19. Hudson A, Cameron C, Matthews J. The wide-scale implementation of a support program for parents of children with an intellectual disability and difficult behaviour. J Intellect Dev Disabil. 2008;33:117-26.

20. Woods D, Catroppa C, Anderson V. Dealing with a head injury in the family (ABI Booklet). Collingwood: Gill Miller Press; 2008.

21. Woods D, Catroppa C, Giallo R, Matthews J, Anderson V. Feasibility and consumer satisfaction ratings following an intervention for families who have a child with acquired brain injury. NeuroRehabilitation. 2012;30:189-98.

22. Woods D, Catroppa C, Godfrey C, Giallo R, Matthews J, Anderson V. Challenging behaviours following paediatric acquired brain injury (ABI): the clinical utility for a manualised behavioural intervention programme. Soc Care Neurodisability. 2014;5:145-59.

23. Woods D, Catroppa C, Godfrey C, Anderson V. Long-term maintenance of treatment effects following intervention for families with children who have acquired brain injury. Soc Care Neurodisability. 2014;5:70-82.

24. Oh H, Rizo C, Enkin M, Jadad A: What is eHealth (3): a systematic review of published definitions. J Med Internet Res. 2005;7:e1. https://www.ncbi.nlm. nih.gov/pmc/articles/PMC1550636/.

25. Wade SL, Wolfe CR, Pestian JP. A web-based family problem-solving intervention for families of children with traumatic brain injury. Behav Res Methods Instrum Comput. 2004;36:261-9.

26. Antonini T, Raj S, Oberjohn K, Wade S. An online positive parenting skills programme for paediatric traumatic brain injury: feasibility and parental satisfaction. J Telemed Telecare. 2012;18:333-8.

27. Wade SL, Carey J, Wolfe CR. An online family intervention to reduce parental distress following pediatric brain injury. J Consult Clin Psychol. 2006;74:445.

28. Rietdijk R, Togher $L$, Power E. Supporting family members of people with traumatic brain injury using telehealth: a systematic review. J Rehabil Med. 2012;44:913-21.

29. Conoley JC, Sheridan SM. Pediatric traumatic brain injury: challenges and interventions for families. J Learn Disabil. 1996;29:662-9.

30. Berry JG, Jamieson LM, Harrison JE. Head and traumatic brain injuries among Australian children, July 2000-June 2006. Inj Prev. 2010;16:198-202.

31. Richardson LK, Christopher Frueh B, Grubaugh AL, Egede L, Elhai JD. Current directions in videoconferencing tele-mental health research. Clin Psychol Sci Pract. 2009;16:323-38

32. Cuevas CDL, Arredondo MT, Cabrera MF, Sulzenbacher H, Meise U. Randomized clinical trial of telepsychiatry through videoconference versus face-to-face conventional psychiatric treatment. Telemed J E-Health. 2006; 12:341-50.

33. Stubbings DR, Rees CS, Roberts LD, Kane RT. Comparing in-person to videoconference-based cognitive behavioral therapy for mood and anxiety disorders: randomized controlled trial. J Med Internet Res. 2013;15:e258.

34. Pesämaa L, Ebeling $H$, Kuusimäki M-L, Winblad I, Isohanni M, Moilanen I. Videoconferencing in child and adolescent telepsychiatry: a systematic review of the literature. J Telemed Telecare. 2004;10:187-92.

35. Taylor HG, Alden J. Age-related differences in outcomes following childhood brain insults: an introduction and overview. J Int Neuropsychol Soc. 1997;3:555-67.

36. Anderson VA, Morse SA, Catroppa C, Haritou F, Rosenfeld JV. Thirty month outcome from early childhood head injury: a prospective analysis of neurobehavioural recovery. Brain. 2004;127:2608-20.

37. Anderson VA, Catroppa C, Haritou F, Morse S, Rosenfeld JV. Identifying factors contributing to child and family outcome 30 months after traumatic brain injury in children. J Neurol Neurosurg Psychiatry. 2005;76:401-8.

38. Anderson VA, Catroppa C, Haritou F, Morse S, Pentland L, Rosenfeld J, Stargatt R. Predictors of acute child and family outcome following traumatic brain injury in children. Pediatr Neurosurg. 2001;34:138-48.

39. Reilly PL, Simpson DA, Sprod R, Thomas L. Assessing the conscious level in infants and young children: a paediatric version of the Glasgow Coma Scale. Childs Nerv Syst. 1988;4:30-3.

40. Teasdale G, Jennett B. Assessment of coma and impaired consciousness: a practical scale. Lancet. 1974;304:81-4.

41. Roberts G, Howard K, Spittle AJ, Brown NC, Anderson PJ, Doyle LW. Rates of early intervention services in very preterm children with developmental disabilities at age 2 years. J Paediatr Child Health. 2008;44:276-80.

42. Achenbach TM, Rescorla LA. Manual for the ASEBA Preschool Forms \& Profiles. Burlington: University of Vermont, Research Center for Children, Youth, and Families; 2000.

43. Miller IW, Epstein NB, Bishop DS, Keitner GI. The McMaster family assessment device: reliability and validity. J Marital Fam Ther. 1985;11:345-56.

44. Epstein NB, Baldwin LM, Bishop DS. The McMaster family assessment device. Marital Fam Ther. 1983:9:171-80.

45. Byles J, Byrne C, Boyle MH, Offord DR. Ontario Child Health Study: reliability and validity of the general functioning subscale of the McMaster Family Assessment Device. Fam Process. 1988;27:97-104.

46. Lovibond S, Lovibond P. Manual for the Depression Anxiety Stress Scales. Sydney: Psychology Foundation of Australia; 1995.

47. Arnold DS, O'Leary SG, Wolff LS, Acker MM. The Parenting Scale: a measure of dysfunctional parenting in discipline situations. Psychol Assess. 1993;5:137. 
48. Johnston C, Mash EJ. A measure of parenting satisfaction and efficacy. J Clin Child Psychol. 1989;18:167-75.

49. Antonini TN, Raj SP, Oberjohn KS, Cassedy A, Makoroff KL, Fouladi M, Wade SL. A pilot randomized trial of an online parenting skills program for pediatric traumatic brain injury: improvements in parenting and child behavior. Behav Ther. 2014;45:455-68.

50. Wade SL, Michaud L, Brown TM. Putting the pieces together: preliminary efficacy of a family problem-solving intervention for children with traumatic brain injury. J Head Trauma Rehabil. 2006;21:57-67.

51. Wade SL, Oberjohn K, Burkhardt A, Greenberg I. Feasibility and preliminary efficacy of a web-based parenting skills program for young children with traumatic brain injury. J Head Trauma Rehabil. 2009;24:239-47.

52. Woods D, Catroppa C, Godfrey C, Giallo R, Matthews JA, Anderson V. A telehealth intervention for families caring for a child with traumatic brain injury (TBI). Soc Care Neurodisability. 2014;5:51-62.

Submit your next manuscript to BioMed Central and we will help you at every step:

- We accept pre-submission inquiries

- Our selector tool helps you to find the most relevant journal

- We provide round the clock customer support

- Convenient online submission

- Thorough peer review

- Inclusion in PubMed and all major indexing services

- Maximum visibility for your research

Submit your manuscript at www.biomedcentral.com/submit
Biomed Central 